\title{
Near-Infrared Resonance Energy Transfer Glucose Biosensors in Hybrid Microcapsule Carriers
}

\author{
Swetha Chinnayelka, ${ }^{1}$ Huiguang Zhu, ${ }^{1}$ and Mike McShane ${ }^{1,2,3}$ \\ ${ }^{1}$ Institute for Micromanufacturing, Louisiana Tech University, Ruston, LA 71272, USA \\ ${ }^{2}$ Biomedical Engineering Program, Louisiana Tech University, Ruston, LA 71272, USA \\ ${ }^{3}$ Department of Biomedical Engineering, Texas A\&M University, 337 Zachry Engineering Center, \\ College Station, TX 77843-3120, USA
}

Correspondence should be addressed to Mike McShane, mcshane@tamu.edu

Received 10 June 2008; Accepted 20 July 2008

Recommended by Igor Medintz

Fluorescence-based sensing systems offer potential for noninvasive monitoring with implantable devices, but require carrier technologies that provide suitable immobilization, accessibility, and biocompatibility. Recent developments towards this goal include a competitive binding assay for glucose that has been encapsulated in semipermeable microcapsule carriers. This paper describes an extension of this work to increase the applicability to in vivo monitoring, wherein two significant developments are described: (1) a near-infrared resonance energy transfer system for transducing glucose concentration, and (2) novel hybrid organic-inorganic crosslinked microcapsules as carriers. The quenching-based assay is a competitive binding (CB) system based on apo-glucose oxidase (AG) as the receptor and dextran as the competitive ligand. The encapsulated quencher-labeled dextran and near infrared donor-labeled glucose receptor showed a stable and reversible response with tunable sensitivity of $1-5 \% / \mathrm{mM}$ over the physiological range, making these transducers attractive for continuous monitoring for biomedical applications.

Copyright (c) 2008 Swetha Chinnayelka et al. This is an open access article distributed under the Creative Commons Attribution License, which permits unrestricted use, distribution, and reproduction in any medium, provided the original work is properly cited.

\section{INTRODUCTION}

It is well known that frequent monitoring of glucose concentrations and appropriate countermeasures can help in achieving euglycemia and minimizing secondary complications of diabetes [1-5]. Optical sensors enabling glucose testing without any need for blood extraction are an attractive alternative to the current standard of painful finger-stick testing procedures [6-8]. In particular, implantable fluorescent sensors, which can be embedded in the highly vascularized dermis $(<1 \mathrm{~mm}$ from the skin surface) and remotely monitored using light, would be an ideal solution [9-11]; such sensing mechanisms have been intensely pursued in recent years using techniques based on fluorescence spectroscopy due to the inherent high sensitivity [10-18]. A viable approach to noninvasive yet specific monitoring of clinical biomarkers would also be useful in the management of a large number of conditions where dosage of therapeutic agents is adjusted based on biomarker levels.
While several approaches for fluorescence-based glucose sensing have been developed using Concanavalin A [12, 19-25] and boronic acid receptors [26-32], their use remains complicated by concerns over toxicity, nonspecific and irreversible binding, and response ranges that do not match physiological levels. We recently developed a novel competitive-binding $(\mathrm{CB})$ assay that employs an inactive form of the enzyme glucose oxidase (apo-GOx) as the glucose-binding protein and uses fluorescence resonance energy transfer (RET) to transduce binding [33]. Apo-GOx is highly selective to $\beta$-D-glucose (5-10X, relative to $\alpha$-Dglucose, D-mannose, and sucrose), suspensions of capsules respond in less than 1 minute to glucose titrations, and the $\mathrm{CB}$ assay is responsive in the clinically relevant $0-30$ millimolar $(\sim 0-600 \mathrm{mg} / \mathrm{dL})$ range $[15,33-35]$. In addition, GOx has been designated as "GRAS," generally regarded as safe [36].

We also developed a carrier system that provides accessibility to the analyte while preventing movement of the molecules outside the region of intended use [15, 37-41]. 
This general concept of entrapping glucose sensing chemistry in biocompatible microspheres is an attractive approach to exploiting the sensitivity of fluorescence spectroscopy and the specificity of molecular recognition agents such as proteins for in vivo use. We pioneered the use of semipermeable polyelectrolyte microcapsules [42-45] for this purpose, because they are particularly well suited for the requirements of multimolecular assays. In this approach, the use of microcapsules enables the free movement of the ligand, receptor, and analyte during the process of competitive displacement, while the semipermeable capsule walls entrap the larger sensing assay elements in the capsule interior, yet allow free glucose transport through capsule walls. The feasibility of using the encapsulated CB assay as a method of glucose monitoring has been demonstrated using multiple RET pairs, including green/orange [15] and orange/red [35] systems; the ability to translate the transduction system to different wavelength regions is particularly advantageous to the current study. The encapsulation was shown to be stable [15] and thus the useful operating lifetime of the microcapsule assays will be determined by photobleaching and protein denaturation kinetics; investigation of these processes, along with quantitative assessment of response time, is currently underway. It is noteworthy that this assay has also recently been demonstrated to function within polyelectrolyte-coated alginate microspheres [46].

While these previous reports represent necessary steps toward a viable monitoring system, a number of additional obstacles to clinical acceptance remain. Three improvements that would help move toward the ultimate goal are an increase in the operating wavelengths, a more facile capsule fabrication and encapsulation process, and a stable capsule formulation. Longer wavelengths will enhance penetration of light through tissue. However, while a large selection of organic dyes and inorganic materials have been identified and used in RET systems [47], the broad excitation spectrum of available NIR dyes makes it difficult to find an efficient RET pair for the NIR region. We note that it is also desirable to match the donor excitation spectrum to the emission of an inexpensive light source, such that low-cost monitoring technology may be employed. The red spectral region is attractive for this purpose, due to the wide availability of excellent red LED and laser diode sources.

These problems were solved in this work by (1) converting the transduction scheme from a system in which the acceptor emits photons to one in which the acceptor is deexcited through nonradiative pathways, where a nearinfrared dye (Alexa Fluor 647) is attached to the glucose receptor and a broadband quencher (QSY21) is attached to the competitive ligand, and (2) encapsulating the assay in hybrid polymer-silica microshells. A number of different microcapsules and construction strategies with controllable shell thickness and properties have been proposed based on the versatile LbL self-assembly process [44, 4851]. Variations of these have been demonstrated for the encapsulation of various macromolecules, such as proteins [39, 52, 53], proteins/polysaccharide complexes [15], and enzymes [39, 41, 54-58]. While several encapsulation methods were based on controlling the permeability of the capsule wall $[51,59,60]$, precipitation techniques $[61,62]$, or polymerization of monomers in microcapsules [41], we have shown that microcapsules with walls comprising photosensitive polymers can be used for highly efficient and stable encapsulation of macromolecules [39]. The disadvantages of this technique, however, include the use of toxic polymers, inconvenient assembly conditions that require protection from UV light prior to UV-initiated crosslinking, and relatively low stability of the polymeric shells during manipulation. While inorganic silica microcapsules and smart organic/inorganic microcapsules have also been proposed $[42,63,64]$, the processes required to realize these are harsh and tedious, requiring exposure to strong acidic/basic conditions, high temperatures, and UV light, all of which pose potential hazards to biological molecules. Therefore, in this work, we explored the possibility of forming hybrid organic/inorganic microcapsules and encapsulating established sensing reagents into the capsules with milder processes. Specifically, we describe the modification of poly(allylamine hydrochloride) (PAH) with glycidylsilane, such that the resulting $\mathrm{PAH}$-silane conjugate could be alternately adsorbed with poly(styrene sulfonate) (PSS), to obtain PSS/PAH-silane microcapsules that slowly crosslink. When the capsules are suspended in a solution containing the macromolecular sensing reagents, the macromolecules diffuse inside and then are trapped as the crosslinking occurs.

This paper describes the investigation of an NIR glucose sensor comprised of competitive binding assay encapsulated in hybrid microcapsules, and compares the performance characteristics with those obtained for the FITC/TRITC and TRITC/Cy5 energy transfer pairs. We also report a novel method for the encapsulation of macromolecules into silanemodified PSS/PAH microcapsules that eventually form organo/inorgano hybrid microcapsules. The hollow silanebased microcapsules slowly form an interpenetrating silica network due to the hydrolysis and subsequent condensation of silane. While the thorough characterization of the hybrid microcapsules will be described in a separate report, the details of the materials and methods used to fabricate these unique structures are provided here for completeness.

\section{Assay description}

A schematic of a glucose sensor based on this quenching mechanism is shown in Figure 1. The dyes used in this sensing mechanism are Alexa Fluor (AF) 647, QSY21, and AF750. AF647 emits in the range of $650-720 \mathrm{~nm}$, which overlaps with the QSY21 absorbance spectrum. Therefore, when AF647 and QSY21 are in close proximity, QSY21 significantly quenches the fluorescence of AF647. As QSY21 is not fluorescent, there is only one fluorescent peak, preventing ratiometric analysis of the data. A second nearinfrared dye (Alexa Fluor 750) is integrated into the walls of the microcapsules, acting as a reference fluorophore for ratiometric monitoring; AF750 is weakly excited at $640 \mathrm{~nm}$ but is not quenched by QSY21. Thus, when apo-GOx tagged to AF647 is exposed to QSY21-dextran (QSY-dex), they will be in close proximity due to the binding affinity between apo-GOx and dextran. This results in low emission intensity 


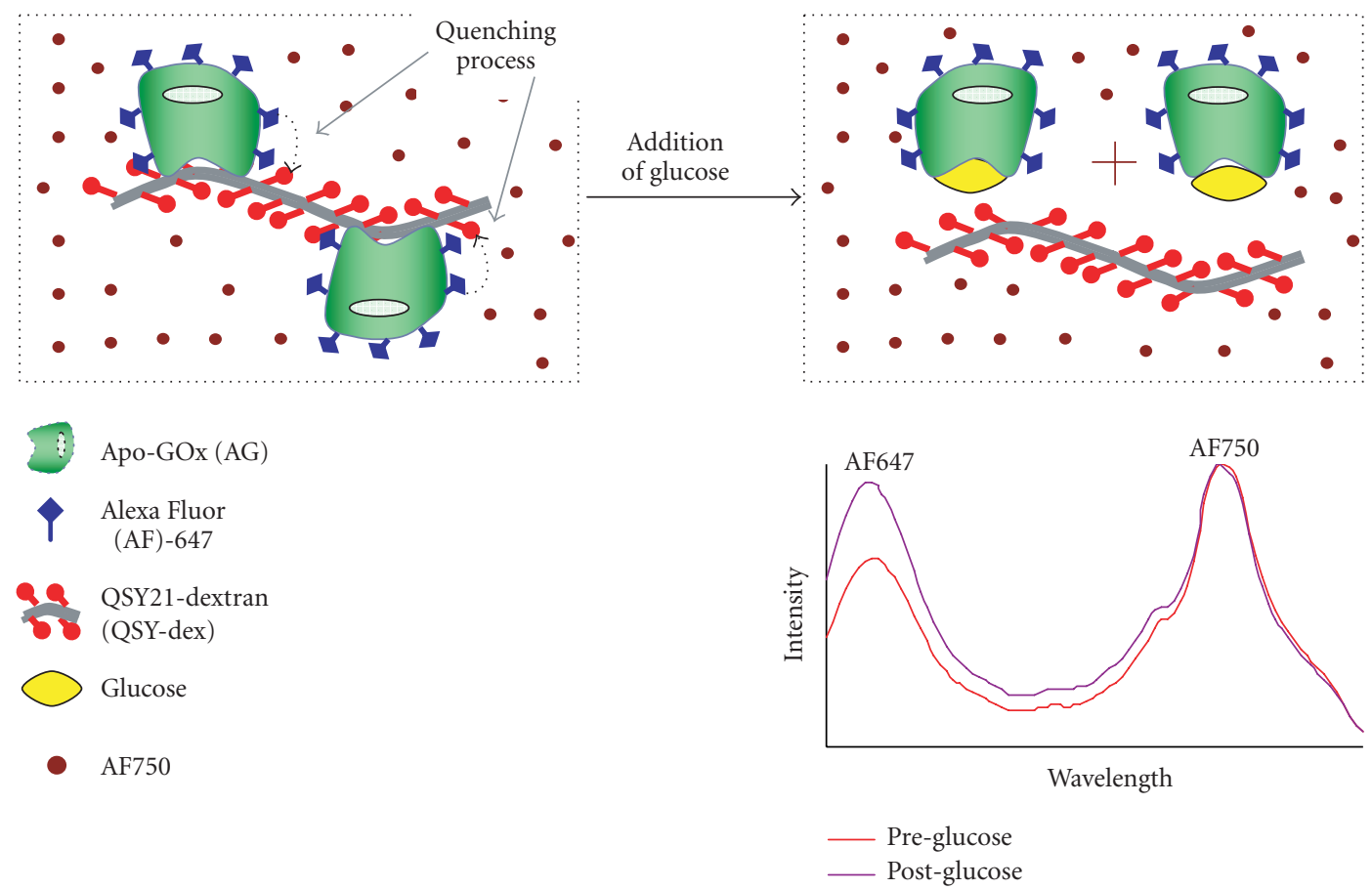

FIGURE 1: Schematic of the RET quenching system for glucose monitoring based on the competition between dextran and glucose for binding sites on apo-GOx.

at $664 \mathrm{~nm}$ due to the quenching of AF647 by QSY21. The addition of glucose results in the displacement of dextran from apo-GOx, decreasing the quenching of AF647 and increasing the emission at $664 \mathrm{~nm}$ (Figure 1). Thus, the glucose sensitivity of the AF647-apo-GOx (AF-AG)/QSY-dex complexes entrapped in AF750-labeled microcapsules can be determined by measuring the changes in fluorescence intensities of AF647 relative to AF750.

\section{Theory}

The homogeneous energy transfer assay described above can be mathematically described by expressions for the competitive binding reactions between the immobilized receptor $(R)$ and ligand $(L)$. The corresponding association constants $\left(K_{1}\right.$ $\left.\& K_{2}\right)$ are given as $L+R \stackrel{K_{1}}{\longrightarrow} L R$, where $K_{1}=[L R] /[L][R]$ and $L R$ is the ligand/receptor complex. Similarly, with analyte $(A)$, the binding reaction is described by $A+R \stackrel{K_{2}}{\longrightarrow} A R$, where $K_{2}=[A R] /[A][R]$ and $A R$ is the analyte/receptor complex. By considering the concentrations of free (unbound) acceptor-labeled receptor, donor-labeled ligand, and analyte, respectively (subscript " $t$ " denotes total concentrations), a final equation to describe the entire system is $[L] /\left[L_{t}\right]-1+$ $R_{t} /\left[L_{t}\right]-1 /[L] K_{1}+1 / K_{1}\left[L_{t}\right]-[A] K_{2} /[L] K_{1}+[A] K_{2} /\left[L_{t}\right] K_{1}=$ 0 , which can also be written in simplified, dimensionless form as: $\left([L] /\left[L_{t}\right]\right)^{2}+\left([L] /\left[L_{t}\right]\right)\left[\left(R_{t} /\left[L_{t}\right]-1\right)+\left([A] K_{2}+\right.\right.$ $\left.1) /\left[L_{t}\right] K_{1}\right]-\left([A] K_{2}+1\right) /\left[L_{t}\right] K_{1}=0$, where $\left([L] /\left[L_{t}\right]\right)$ and $\left(\left([A] K_{2}+1\right) /\left(\left[L_{t}\right] K_{1}\right)\right)$ give the ratio of free to total ligand and dimensionless analyte concentration, respectively [65]. Dimensionless analyte concentration depends upon the ana- lyte concentration and receptor-analyte affinity compared to the concentration and affinity of the competing ligand. Since the amount of unquenched receptor ( $\left.R_{\text {unquenched }}\right)$ is of interest in this study, this quantity can be found as the sum of the free receptor and analyte-bound receptor concentrations $[R]+[A R]=\left[R_{t}\right]-[L R]$. This allows solving for the measured quantity, relative amount of fluorescent receptor $([R]+[A R]) /\left[R_{t}\right]=1-\left([L R] /\left[R_{t}\right]\right)=1-\left(\left[L_{t}\right] /\left[R_{t}\right]\right)(1-$ $\left.L / L_{t}\right)$.

A key aspect of this system is the dependence of the response on different concentrations of assay components, in both absolute and relative senses. Using the equations above, the graphs in Figure 2 were produced to illustrate the effect of these parameters. Clearly, the response shifts dramatically when the ratios of ligand to receptor or absolute reagent concentrations are altered; specifically, as $\left[L_{t}\right] /\left[R_{t}\right]$ is increased, the predicted response magnitude increases while responding over the same range. With dropping $\left[L_{t}\right] /\left[R_{t}\right]$ values, the response range shifts to lower concentrations and a loss of sensitivity is observed, due to the incompletely quenched receptor fluorescence in the absence of analyte resulting from insufficient quenching ligand. Furthermore, the range over which the response is observed varies directly with absolute assay concentration. From these models, the concentrations of the assay elements needed for any application can be estimated based on the required detection range if association constants are known, and the effects of inhomogeneity in capsule populations on the sensor response can be predicted.

The response of the sensing system can be characterized by the effective dissociation constant of the sensing assay, 




(a)

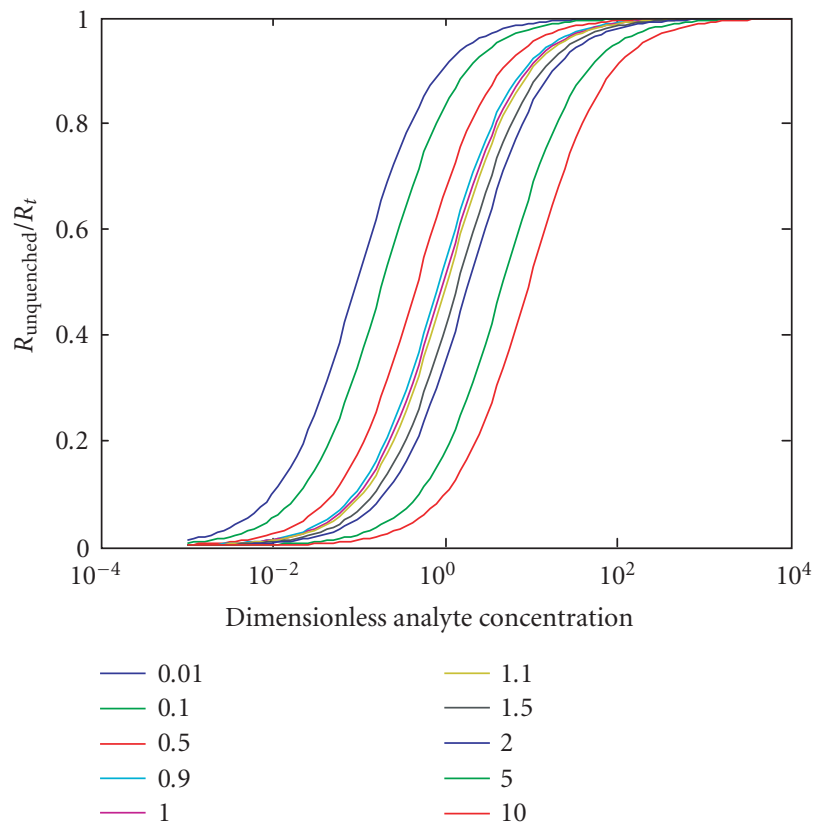

(b)

FIGURE 2: Effect of dimensionless analyte concentration on relative response $\left(R_{\text {unquenched }} / R_{t}\right)$ of a generic quenching-based competitivebinding assay, for varying ligand/receptor ratio $\left(L_{t} / R_{t}\right),((\mathrm{a})$ ratio values given in legend $)$ and total assay concentration $\left((\mathrm{b}) L_{t} / R_{t}=10\right.$, legend gives values for multiplier).

also referred to as the concentration at which half of the full response is observed $\left(C_{g}(S=1 / 2)\right)$ [66]. Clearly, this quantity shifts to larger values with increasing assay concentration. To predict the effects of increasing assay concentration on the response to analyte binding, realistic values for ligand and receptor concentrations and their respective association constants were used $\left(R_{t}=42 \mathrm{nM} ; L_{t}=\right.$ $140 \mathrm{nM} ; K_{L}=K_{1}=10^{7} \mathrm{M}^{-1} ; K_{A}=K_{2}=30.3 \mathrm{M}^{-1}$ ) to calculate the percentage change in unquenched receptor concentration versus analyte concentration. It is even more obvious from this linear plot (see Figure 3 ) that the magnitude of the response is greatly enhanced with an increase in assay concentration, and the range over which the response changes appreciably due to additional analyte increases as well.

\section{EXPERIMENTAL DETAILS}

\subsection{Materials}

Glycidyl 3-(trimethoxysilyl)propyl ether (glycidyl-silane), GOx (G-2133), sodium poly(styrene sulfonate) (PSS, $\mathrm{MW} \sim 1 \mathrm{MDa}$ ), poly(allylamine hydrochloride) (PAH, MW $70 \mathrm{kDa}), \beta$-D-glucose, sodium bicarbonate, dimethyl formamide, ammonium sulfate, and sodium acetate buffer were obtained from Sigma. Succinimidyl ester forms of Alexa Fluor 647 (AF647), Alexa Fluor 750 (AF750), and QSY 21 (QSY21) (Invitrogen) were used to label apo-GOx, $\mathrm{PAH}$, and amino-dextran (500 kDa, Invitrogen), respectively, using a standard amine labeling procedure (Invitrogen). All reagents were used as received. $\mathrm{MnCO}_{3}(5 \mu \mathrm{m})$ particles were prepared as previously described [40].

\subsection{Instrumentation}

A UV-Vis spectrometer (Perkin Elmer Lambda 45) was used to collect absorbance spectra. The slit size $(4 \mathrm{~nm})$ and scanning speed $(480 \mathrm{~nm} / \mathrm{min})$ were held constant throughout all the experiments. A scanning fluorescence spectrometer (QM1, Photon Technology International) with an extendedwavelength PMT (R928) was used to collect fluorescence emission spectra while exciting the sample at $640 \mathrm{~nm}$. Confocal images were taken with a Leica TCS SP2 microscope equipped with a $63 \mathrm{X}$ oil immersion objective and a red $\mathrm{He}-\mathrm{Ne}$ excitation laser. Counts and sizes of microcapsules were obtained with a Beckman Coulter counter (Z2) using a $100 \mu \mathrm{m}$ aperture. The assembly of polyelectrolyte multilayers on colloidal templates was monitored on template particles by measuring the surface potential after the deposition of each polyion; electrophoretic mobility measurements were performed using a ZetaPlus Zeta Potential Analyzer (Brookhaven Instrument Corp.).

\subsection{Methods}

\subsubsection{Solution-phase experiments}

\section{Assay optimization}

The glucose assay concentration was optimized by performing a simple titration experiment to obtain maximum change in signal for a given analyte concentration. The 


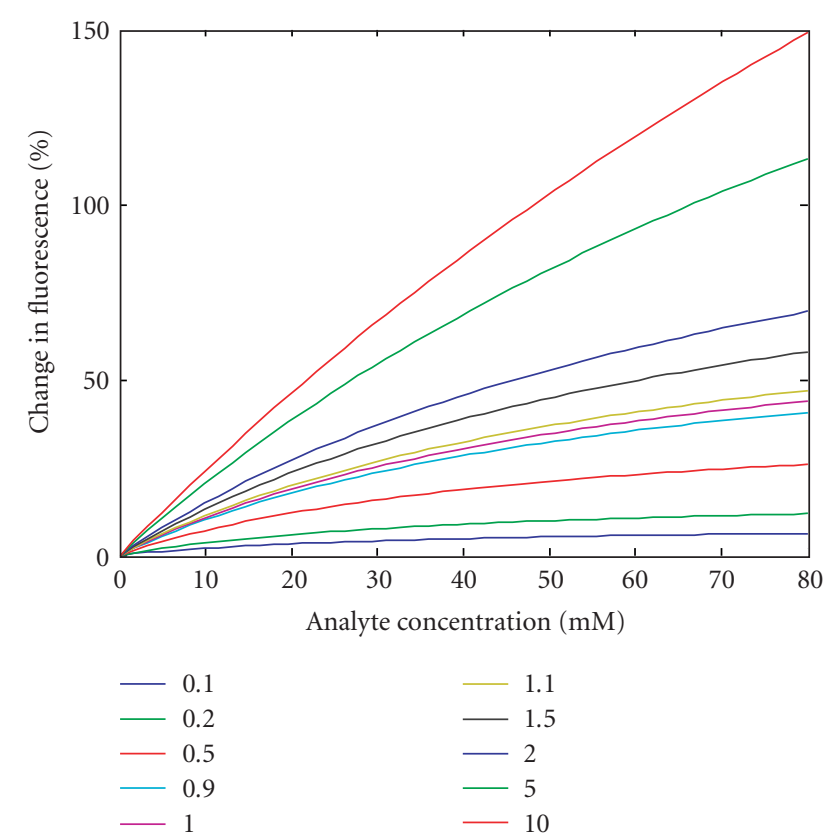

Figure 3: Estimated assay response to analyte, in terms of percentage increase in unquenched receptor. Inset gives value for multiplier used in set the concentration of assay (relative to $R_{t}=$ $42 \mathrm{nM}$ and $L_{t}=140 \mathrm{nM}$ ).

concentrations of the sensing assay elements were optimized based on the concentration-dependent quenching of AF647 by QSY21. To observe the quenching behavior and confirm RET, aliquots of $700 \mathrm{nM}$ QSY21-dextran (QSY-dex) solution were titrated into 17.5, 35, and 70 nM AF647-AG (AFAG) in a stepwise manner, followed by measurements of fluorescence emission.

\section{Glucose sensitivity}

The apo-GOx and amino-dextran used in all experiments were labeled with AF647 and QSY21 at ratios of 3.2 and 2.4, respectively. The quenching process between AF-AG and QSY-dex was monitored using the fluorescence spectrometer by exciting the sample at $640 \mathrm{~nm}$ and collecting the emission across the range of $650-750 \mathrm{~nm}$. Initially, $\sim 16.5$ picomoles of AF647-AG were added to $0.4 \mathrm{~mL}$ of DI water. Based on quenching studies, the initial concentration of QSYdex for $41 \mathrm{nM}$ AF-AG was selected to be $143 \mathrm{nM}$. To assess the relative affinity of apo-GOx for glucose and QSY-dex, recovery in the AF647 emission was observed during the stepwise addition of aliquots of $500 \mathrm{mM} \beta$-Dglucose solution into the sample containing QSY-dex/AFAG complexes. The effect of total QSY-dex concentration on the displacement behavior was investigated by repeating the same procedure at different concentrations of QSYdex. Fluorescence changes in AF647 peak were corrected for dilution effects by titrating QSY-dex/AF-AG complexes with DI water in parallel experiments, then subtracting the change due to water from that due to glucose. For all the experiments, sensitivity curves were constructed by plotting the dilution-corrected emission intensity at $664 \mathrm{~nm}$ versus glucose concentration.

\subsubsection{Fabrication of hybrid microcapsules and encapsulation of sensing reagents}

\section{Microcapsule fabrication}

The PSS and PAH solutions used for building multilayer films were prepared in DI water at $50 \mathrm{mM}$ (concentration based on monomer). For LbL assembly of PSS/PAH-silane, $20 \mu \mathrm{L}$ of glycidyl-silane was added into $1 \mathrm{~mL}$ of PAH solution. Multilayer films of PSS/PAH-silane films were assembled on the surface of $5 \mu \mathrm{m} \mathrm{MnCO} 3$ particles (Figures 4(a) and $4(\mathrm{~b})$ ) by suspending the particles in polymer solutions for 15 minutes, followed by three centrifugation and rinse steps to remove unbound polymer [40]. The film architecture on the surface of the templates at the completion of the assembly was (PSS/(PAH-silane) $)_{4} / \mathrm{PSS}$. To confirm charge reversal, surface potential measurements were completed after deposition of each polymer layer. Following the completion of polymer layer deposition, $\mathrm{MnCO}_{3}$ core particles were dissolved (Figure 4(c)), yielding $5 \mu \mathrm{m}$ inner diameter silanemodified hollow microcapsules.

\section{Loading of sensing reagents}

Microcapsules with the (PSS/(PAH-silane) $)_{4} / \mathrm{PSS}$ architecture were dispersed in a mixture of AF-AG and QSY-dex (230 and $990 \mathrm{pM}$, resp.) (Figure 4(d)). The microcapsules were incubated in the loading solution for two days to allow sufficient time for the hydrolysis and condensation of silane, facilitating the formation of a denser and less permeable interpenetrating network (Figure 4(e)). The capsules were rinsed in DI water to remove unencapsulated assay molecules, yielding microcapsules loaded with AF647AG and QSY21-dextran (Figure 4(f)). Finally, the reference dye was incorporated into the microcapsule walls by coating the outer layer (PSS) of microcapsules with PAHAF750 via electrostatic self-assembly. Confocal microscopy was then used to assess the loading of assay elements into microcapsules. Leaching of the loaded assay elements was determined by measuring absorbance and fluorescence from capsule supernatant five weeks after encapsulation. A standard solution of $50 \mathrm{nM}$ AF-AG was used to estimate the supernatant concentration as a percentage of a standard.

\subsubsection{Encapsulated sensor response tests}

\section{Sensor response in microcapsules}

The AF750-labeled microcapsules loaded with AF-AG and QSY-dex were suspended in DI water, and an initial fluorescence spectrum was collected. Glucose solution $(500 \mathrm{mM})$ was then titrated into the microcapsule suspension, as was performed for the solution-phase studies. The change in the relative emission intensities at 664 and $780 \mathrm{~nm}$ was calculated for each spectrum and plotted with respect to glucose concentration. To confirm theoretical prediction on the effect of receptor/competitive ligand concentration and 


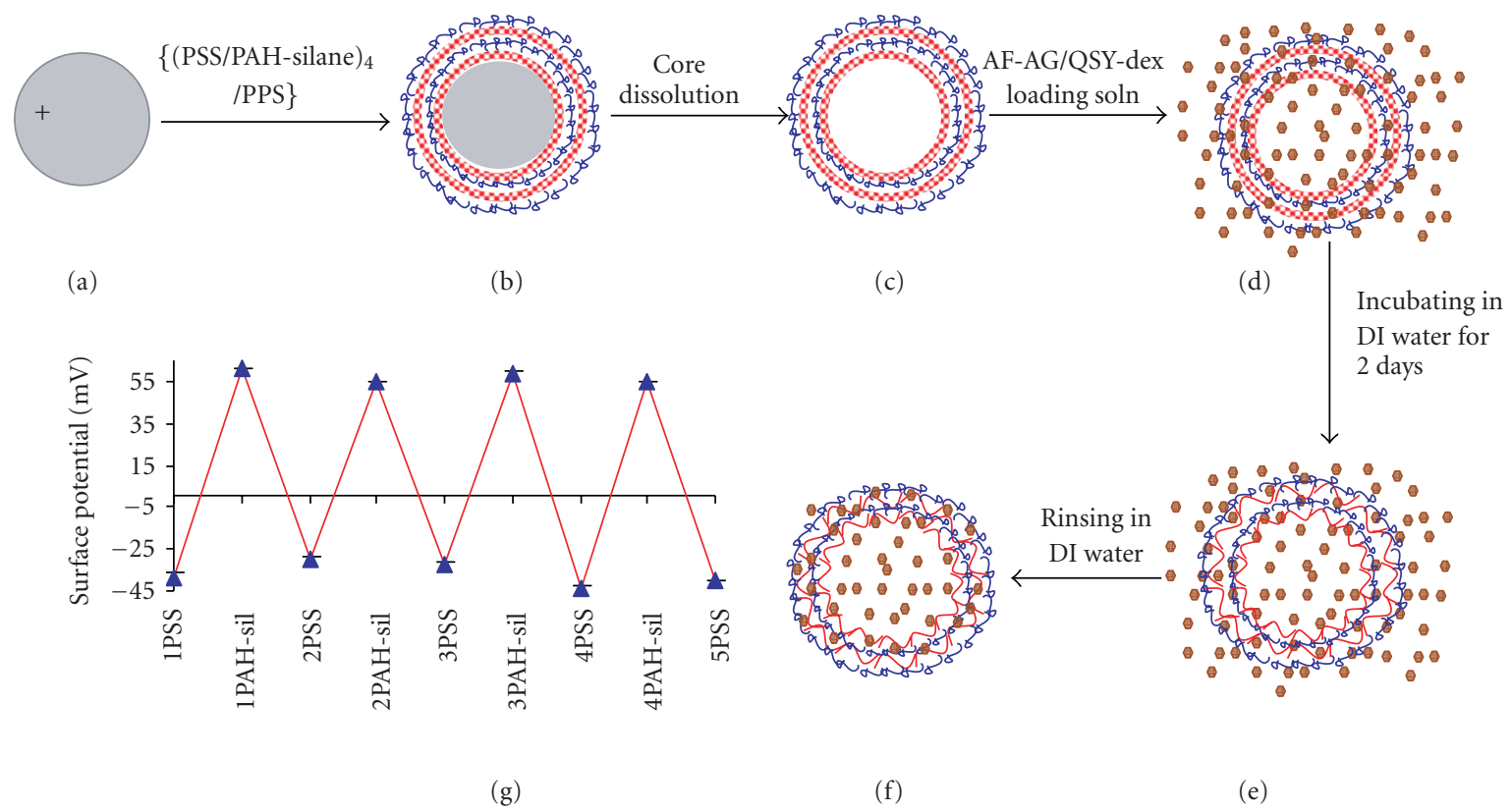

FIGURE 4: Process for organic/inorganic hybrid microcapsule fabrication and loading (a)-(c); encapsulation of assay reagents into hollow microcapsules (d)-(f); and (g) surface potential values obtained after coating each layer on $\mathrm{MnCO}_{3}$ particles.



FIGURE 5: Quenching of AF647-AG in response to QSY21-dextran titration.

to evaluate the possibility of tailoring the sensor response, these experiments were repeated with four different concentrations of capsules, $\left(4.3 \times 10^{5}, 8.6 \times 10^{5}, 1.72 \times 10^{6}\right.$, $3.87 \times 10^{6}$ capsules $/ \mathrm{mL}$ ). All experiments were repeated three times, and calculated changes in ratio values were pooled for statistical analysis.

\section{Reversibility}

To test the reversibility of the microcapsule sensors, exposure to glucose in random order with respect to concentration was performed. To achieve this, microcapsules were dispersed in DI water $\left(2.88 \times 10^{6}\right.$ capsules $\left./ \mathrm{mL}\right)$ and the glucose concentration of the suspension was increased by addition of glucose stock solution. The glucose level was decreased by soaking the capsules in DI water to remove the glucose after each measurement. In each case, after adding glucose to the desired concentration and measuring the fluorescence, the suspension was centrifuged three times, the supernatant (glucose solution) was removed, and an equal volume of fresh DI water was added. The change in AF647/AF740 peak ratio was obtained at each step by collecting a fluorescence scan. This procedure was repeated to cover the concentration range of $0-60 \mathrm{mM}$, in the order of $0,5.3,0,13.5,20,3.31$, 26.6, 32.5, 5.5, 44.6, 6.3, 61.6, $10 \mathrm{mM}$. The experiment was repeated three times, and results pooled for statistical analysis.

\section{RESULTS AND DISCUSSION}

\subsection{Solution phase experiments}

\section{Assay optimization}

AF-AG samples exhibited decreasing emission intensity during the titration of QSY-dex, following a pattern predicted by equilibrium binding (Figure 5). This suggests that the quenching is due in part to RET and possibly also due to radiative processes. By fitting the data collected from three experiments performed at different concentrations of AF$\mathrm{AG}$, the $k_{d}$ for QSY21-dextran was estimated to be between 50 and $100 \mathrm{nM}$. These findings prove the efficiency of the RET-based quenching process due to apo-GOx:dextran association and provide insight into the binding affinity, which is useful in assay design. 


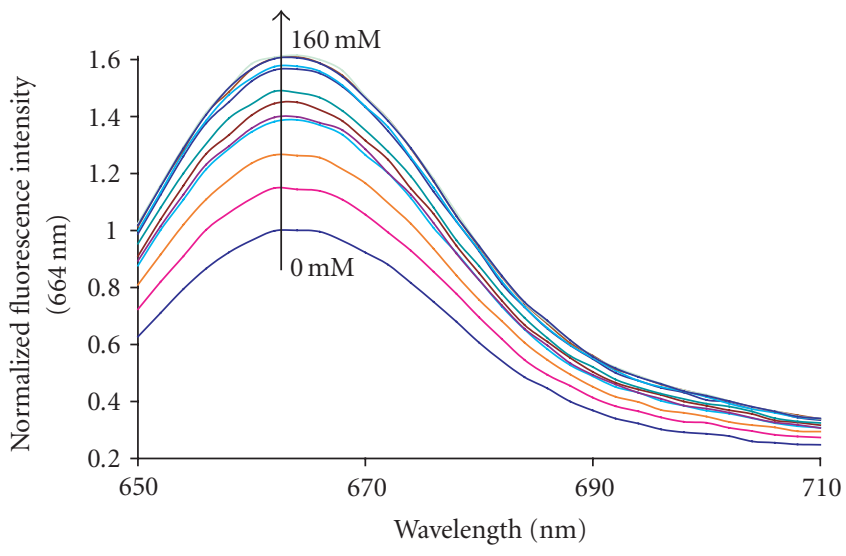

a)

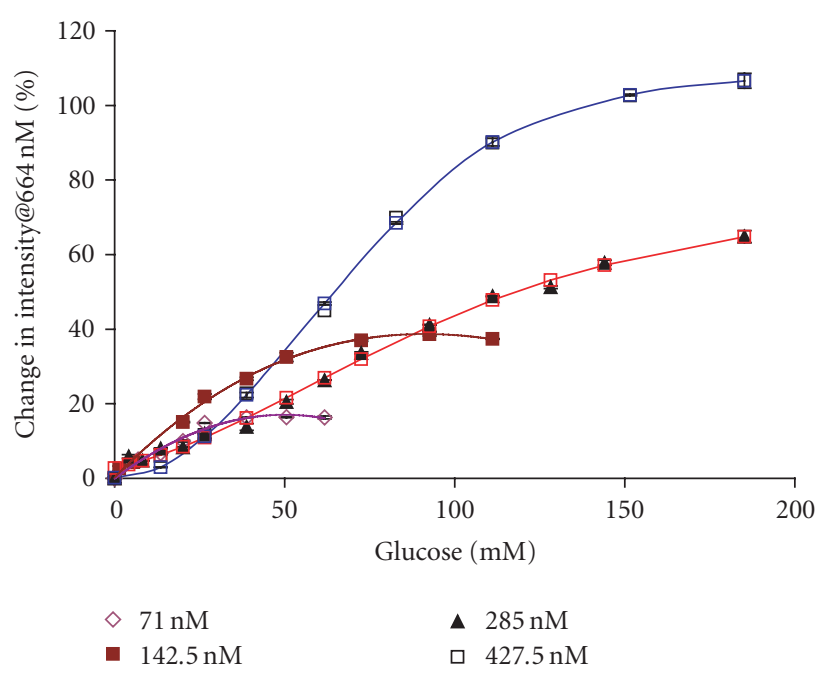

(b)

FIGURE 6: Normalized fluorescence spectra (a) and percentage change in AF647 emission with the addition of glucose into AF-AG/QSY-dex complexes (b). Lines are regression curves used to identify trends. Error bars indicate one standard deviation of three replicate measurements. The inset of (b) indicates the QSY21-dex concentrations.

\section{Glucose response tests}

Taking the optimum assay concentrations obtained from the quenching experiments into consideration, an assay mixture containing $142 \mathrm{nM}$ QSY-dex and $42 \mathrm{nM}$ AF-AG was prepared in DI water and subjected to sequential glucose additions. As hypothesized, the added glucose displaced dextran from apoGOx, resulting in decreased quenching and correspondingly enhanced fluorescence emission at $664 \mathrm{~nm}$ (Figure 6(a)). Thus, the shift of the assay to longer wavelengths via the RETbased transduction was successful, and the sensitivity of the response $(0.7 \% / \mathrm{mM})$ is comparable to the original system that used visible dyes [33]. The dilution-corrected emission peak ratio changed a total of $25 \%$ (Figure $6(\mathrm{~b})$ ), with a linear response in the physiological range of $0-30 \mathrm{mM}$. Figure 6(b) also contains response curves for varying apo-GOx:dextran combinations, confirming the change in sensitivity and range for different receptor and competitive ligand concentrations that was predicted by theory (Figures 2 and 3 ). These findings prove that the glucose sensitivity of the assay can be varied over a wide concentration range $(0-200 \mathrm{mM})$ by controlling the quencher concentration; however, it is noteworthy that the sensitivity in the region of interest $(0-30 \mathrm{mM})$ is similar in all the experiments, because the same relative number of quencher molecules are displaced due to the same glucose concentration. Thus, the main parameter that can be varied by controlling quencher concentration is the effective dissociation constant of the sensing assay, also referred to as the concentration at which half of the full response is observed $\left(C_{g}(S=1 / 2)\right)$ [66]. In these experiments, the $C_{g}(S=1 / 2)$ was estimated to be $17,25,50$, and $83 \mathrm{mM}$ for the assays with 71, 142.5, 285, and 427.5 nM QSY21-dex, respectively.

\section{Encapsulation of AF-AG/QSY-dex complexes in microcapsules}

The surface potential of microparticles alternated with the addition of each layer, (Figure $4(\mathrm{~g})$ ). This demonstrates the successful fabrication of the shell on the template particles. Microcapsules with (PSS/PAH-silane) ${ }_{4}$ PSS shell architecture were prepared by core dissolution and used for encapsulating AF-AG/QSY-dex complexes, followed by incorporation of the reference dye (AF750). Postloading supernatant concentrations and capsule counts were used to calculate encapsulation amounts of $1 \times 10^{-16}$ and $1.34 \times$ $10^{-16} \mathrm{moles} / \mathrm{capsule}$ for AF-AG and QSY-dex, respectively. Release of AF-AG into supernatant over five weeks was only $0.12 \%$, which represents a 40 -fold decrease in leaching over previous approaches and indicates a stable entrapment of the receptor and competing ligand. By confocal microscopy and titration experiments, the fraction of apo-GOx in the capsule interior was estimated to be $27 \%$ of the total, while the other $73 \%$ was immobilized in the capsule walls and believed to be unable to respond to glucose changes. It will be desirable to improve the encapsulation process to remove unwanted background signals and avoid waste; we believe this may be accomplished by, for example, "presaturating" the nonspecific binding sites in the walls of the capsules with albumin or other blocking reagent. However, we acknowledge that this may raise other difficulties and will leave solution of this problem to future studies.

\section{Sensor response to glucose addition in microcapsules}

The intensity of fluorescence at $664 \mathrm{~nm}$ increased with each addition of $\beta$-D-glucose solution to the microcapsules loaded with AF-AG/QSY-dex complexes, as shown in 


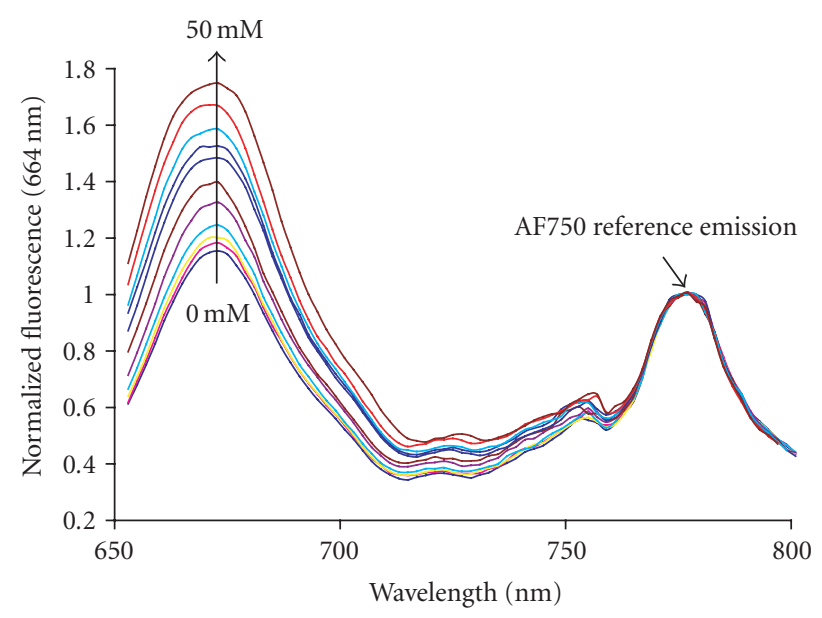

(a)



$$
\begin{aligned}
& \text { Capsules/mL } \\
& 4.3 E+05 \\
& 1.72 E+06
\end{aligned}
$$

$8.6 E+05$

А $3.87 E+06$

(b)

FIGURE 7: Fluorescence spectra of the microcapsules loaded with sensing assay and reference dye (AF750) with the addition of glucose solution. (a) All the spectra are normalized to the reference peak at $776 \mathrm{~nm}$. (b) Relative change in AF647:AF750 peak ratio with the addition of glucose solution. Error bars indicate one standard deviation of three replicate measurements.

TABLE 1: Loading concentration and response properties for samples with varying capsule concentration.

\begin{tabular}{lccccc}
\hline Capsules $/ \mathrm{mL}$ & QSY-dex $($ picomol $)$ & AF-AG $($ picomol $)$ & Sensitivity $(\% \Delta$ ratio/mM $)$ & Linear range $(\mathrm{mM})$ & $C_{g}(S=1 / 2)$ \\
\hline $4.3 \times 10^{5}$ & 18 & 23 & 5.15 & $0-10$ & $0-30$ \\
$8.6 \times 10^{5}$ & 37 & 46 & 3.09 & $0-40$ & 10 \\
$1.72 \times 10^{6}$ & 73 & 92 & 1.97 & $0-80$ & 20 \\
$3.87 \times 10^{6}$ & 184 & 146 & 0.8 & 60 \\
\hline
\end{tabular}

Figure $7(\mathrm{a})$. The corresponding percentage change in the normalized intensity at $664 \mathrm{~nm}$ was calculated and plotted versus glucose concentration, as shown in Figure 7(b). Again, there was no significant drop in sensitivity over the region of interest $(0-30 \mathrm{mM})$ when compared to the visible dye systems $[15,35]$. The decreased sensitivity with increased capsule concentration matches the theory and solutionphase measurements, confirming that the sensitivity can be tailored by changing the competitive binding assay concentration. There was an increase in the sensitivity when comparing microcapsules to the solution-phase response for comparable assay concentrations, on the order of two fold. This is the result of the low concentration of mobile sensing assay elements, due in large part to the entrapment of $\sim 72 \%$ of the encapsulated molecules in the capsule walls. The immobilized apo-GOx and dextran molecules may not dissociate in the presence of glucose molecules, and therefore contribute only a constant background signal to the emission spectrum.

We note that this behavior is consistent with previous studies with visible dyes $[15,35]$, though the total amount and relative fraction of "available" assay reagents is different due to the different capsule structure. Specifically, the current system encapsulates $\sim 10 \mathrm{X}$ the amount of apo-GOx per capsule compared to previous systems, which were more efficient ( $\sim 50 \%$ inside/50\% in walls) [15]; so, even with the apparently low efficiency, a larger loading was accomplished with the new approach. This is further supported by the relative "saturation" point for comparable numbers of microcapsules: for the new system, this is reached around $35 \mathrm{mM}$ for $2 \times 10^{6}$ capsules, while for the previous versions, this was around $20 \mathrm{mM}$ for the same number [15].

Given that physiological sensing is the goal, and that pathophysiological glucose levels experienced by diabetics may range from over $0-30 \mathrm{mM}(0-600 \mathrm{mg} / \mathrm{dL})$, matching the sensor response to this range requires proper selection of reagent concentration. As can be seen in Figure 7 and Table 1, the range of response can be tuned to match the application by controlling the amount of receptor and competing ligand in the sample. For example, to match the $0-30 \mathrm{mM}$ range with a linear response, $37 \mathrm{pmol}$ QSY-dex and $46 \mathrm{pmol}$ AF-AG should be encapsulated.

\subsection{Demonstration of reversible sensor response}

Exposing the microcapsules $\left(2.88 \times 10^{6}\right.$ capsules $\left./ \mathrm{mL}\right)$ to random glucose concentrations confirmed the completely reversible nature of the assay without any loss in sensitivity. 


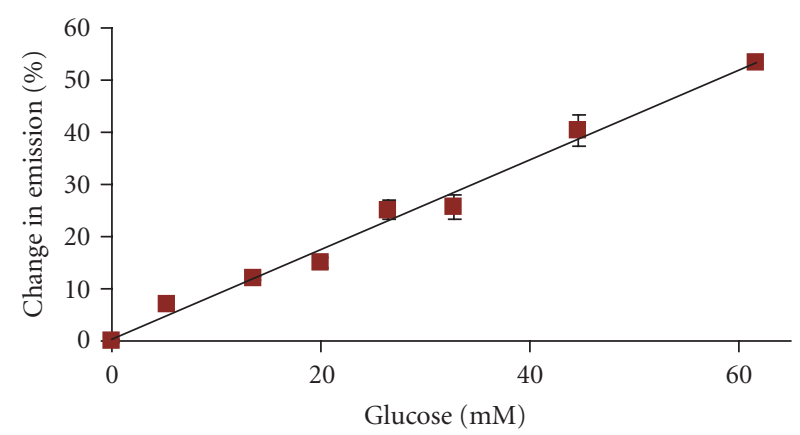

Figure 8: Percentage change in AF647:AF750 peak ratio with the addition of random $\beta$-D-glucose concentrations to the AFAG/QSY-dex-loaded microcapsules. A regression line identifies the linear trend of the response. Error bars indicate one standard deviation of three replicate measurements.

The changes in relative intensity plotted in Figure 8 also illustrate the consistency and linearity of the response over a wide concentration range. The $0.83 \% / \mathrm{mM}$ sensitivity matches that observed for the same concentration of capsules exposed to a stepwise increase in glucose concentrations (Figure 7, Table 1).

\section{CONCLUSIONS}

An efficient RET quenching approach was developed as the transduction mechanism for a dextran/apo-GOx competitive binding assay, extending the operating region into the near-infrared. The sensing assay elements (a quencherlabeled dextran, fluorophore-tagged apo-glucose oxidase, and a fluorescent reference dye) were entrapped in microcapsules using a facile and efficient silane-based encapsulation procedure. Microcapsules containing labeled dextran/apoGOx complexes showed glucose sensitivity of $\sim 1-5 \% / \mathrm{mM}$, which is comparable to the original assay operating in the visible region and is among the most sensitive ever reported. Thus, this system is superior to previous iterations, as it possesses the following desirable qualities: (1) the receptor is nontoxic [36], (2) the silane-based encapsulation procedure employed in this system is simple and free of toxic materials; (3) the sensitivity of this system is equivalent or superior to previous embodiments of the microcapsulebased glucose sensors; and (4) a significant increase in the signal levels (signal to noise ratio) will be obtained when used in biological media, due to decreased scattering at the longer wavelengths of light. These improvements make this system for glucose sensing even more attractive as an implantable technology for continuous glucose monitoring.

\section{ACKNOWLEDGMENTS}

The authors gratefully acknowledge the National Science Foundation (NIRT Grant no. 0210298) and NIH (R01EB000739). Any opinions, findings, and conclusions/recommendations expressed in this material are those of the authors and do not necessarily reflect the view of the National Science Foundation.

\section{REFERENCES}

[1] R. E. Ratner, "Diabetes control and complications. Where do we go from here?" The Online Journal of Current Clinical Trials, vol. 2, doc no. 68, 1993.

[2] J. V. Santiago, "Lessons from the diabetes control and complications trial," Diabetes, vol. 42, no. 11, pp. 1549-1554, 1993.

[3] N. H. White, P. A. Cleary, W. Dahms, D. Goldstein, J. Malone, and W. V. Tamborlane, "Beneficial effects of intensive therapy of diabetes during adolescence: outcomes after the conclusion of the Diabetes Control and Complications Trial (DCCT)," The Journal of Pediatrics, vol. 139, no. 6, pp. 804-812, 2001.

[4] M. Hanefeld and T. Temelkova-Kurktschiev, "Control of postprandial hyperglycemia-an essential part of good diabetes treatment and prevention of cardiovascular complications," Nutrition, Metabolism \& Cardiovascular Diseases, vol. 12, no. 2, pp. 98-107, 2002.

[5] R. E. Carter, D. T. Lackland, P. A. Cleary, et al., "Intensive treatment of diabetes is associated with a reduced rate of peripheral arterial calcification in the diabetes control and complications trial," Diabetes Care, vol. 30, no. 10, pp. 26462648, 2007.

[6] S. Nagl and O. S. Wolfbeis, "Optical multiple chemical sensing: status and current challenges," The Analyst, vol. 132, no. 6, pp. 507-511, 2007.

[7] M. V. Loschenov, A. S. Parfenov, G. L. Kisselev, A. A. Stratonnikov, K. Ershova, and R. W. Steiner, "Fluorescence method for monitoring of glucose in interstitial fluids," in Optical Diagnostics and Sensing of Biological Fluids and Glucose and Cholesterol Monitoring, vol. 4263 of Proceedings of SPIE, pp. 34-39, San Jose, Calif, USA, January 2001.

[8] R. J. McNichols and G. L. Coté, "Optical glucose sensing in biological fluids: an overview," Journal of Biomedical Optics, vol. 5, no. 1, pp. 5-16, 2000.

[9] M. J. McShane, R. J. Russell, M. V. Pishko, and G. L. Coté, "Glucose monitoring using implanted fluorescent microspheres," IEEE Engineering in Medicine and Biology Magazine, vol. 19 , no. 6 , pp. 36-45, 2000.

[10] M. J. McShane, "Potential for glucose monitoring with nanoengineered fluorescent biosensors," Diabetes Technology \& Therapeutics, vol. 4, no. 4, pp. 533-538, 2002.

[11] M. J. McShane, "Microcapsules as "smart tattoo" glucose sensors: engineering systems with enzymes and glucosebinding sensing elements," in Glucose Sensing, J. R. Lakowicz and C. D. Geddes, Eds., vol. 11 of Topics in Fluorescence Spectroscopy, pp. 131-161, Springer, New York, NY, USA, 2006.

[12] R. J. Russell, M. V. Pishko, C. C. Gefrides, M. J. McShane, and G. L. Coté, "A fluorescence-based glucose biosensor using concanavalin A and dextran encapsulated in a poly(ethylene glycol) hydrogel," Analytical Chemistry, vol. 71, no. 15, pp. 3126-3132, 1999.

[13] R. Ballerstadt and J. S. Schultz, "A fluorescence affinity hollow fiber sensor for continuous transdermal glucose monitoring," Analytical Chemistry, vol. 72, no. 17, pp. 4185-4192, 2000.

[14] M. J. McShane, D. P. O’Neal, R. J. Russell, M. V. Pishko, and G. L. Coté, "Progress toward implantable fluorescence-based sensors for monitoring glucose levels in interstitial fluid," in Optical Diagnostics of Biological Fluids V, vol. 3923 of Proceedings of SPIE, pp. 78-87, San Jose, Calif, USA, January 2000.

[15] S. Chinnayelka and M. J. McShane, "Microcapsule biosensors using competitive binding resonance energy transfer assays 
based on apoenzymes," Analytical Chemistry, vol. 77, no. 17, pp. 5501-5511, 2005.

[16] J. Q. Brown and M. J. McShane, "Modeling of spherical fluorescent glucose microsensor systems: design of enzymatic smart tattoos," Biosensors and Bioelectronics, vol. 21, no. 9, pp. 1760-1769, 2006.

[17] S. R. Nayak and M. J. McShane, "Fluorescence glucose monitoring based on transduction of enzymatically-driven pH changes within microcapsules," Sensor Letters, vol. 4, no. 4, pp. 433-439, 2006.

[18] E. W. Stein, P. S. Grant, H. Zhu, and M. J. McShane, "Microscale enzymatic optical biosensors using mass transport limiting nanofilms. 1. Fabrication and characterization using glucose as a model analyte," Analytical Chemistry, vol. 79, no. 4, pp. 1339-1348, 2007.

[19] J. S. Schultz and G. Sims, "Affinity sensors for individual metabolites," Biotechnology Bioengineering Symposium, no. 9, pp. 65-71, 1979.

[20] D. L. Meadows and J. S. Schultz, "Miniature fiber optic sensor based on fluorescence energy transfer," in Fiber Optic Medical and Fluorescent Sensors and Applications, vol. 1648 of Proceedings of SPIE, pp. 202-211, Los Angeles, Calif, USA, January 1992.

[21] R. Ballerstadt, A. Gowda, and R. McNichols, "Fluorescence resonance energy transfer-based near-infrared fluorescence sensor for glucose monitoring," Diabetes Technology \& Therapeutics, vol. 6, no. 2, pp. 191-200, 2004.

[22] J. C. Pickup, F. Hussain, N. D. Evans, and N. Sachedina, "In vivo glucose monitoring: the clinical reality and the promise," Biosensors and Bioelectronics, vol. 20, no. 10, pp. 1897-1902, 2005.

[23] R. Ballerstadt, C. Evans, R. McNichols, and A. Gowda, "Concanavalin A for in vivo glucose sensing: a biotoxicity review," Biosensors and Bioelectronics, vol. 22, no. 2, pp. 275284, 2006.

[24] K.-C. Liao, T. Hogen-Esch, F. J. Richmond, L. Marcu, W. Clifton, and G. E. Loeb, "Percutaneous fiber-optic sensor for chronic glucose monitoring in vivo," Biosensors and Bioelectronics, vol. 23, no. 10, pp. 1458-1465, 2008.

[25] K. Y. Cheung, W. C. Mak, and D. Trau, "Reusable optical bioassay platform with permeability-controlled hydrogel pads for selective saccharide detection," Analytica Chimica Acta, vol. 607, no. 2, pp. 204-210, 2008.

[26] C. R. Cooper and T. D. James, "Fluorescent sensors based on boronic acids," in Advances in Fluorescence Sensing Technology IV, vol. 3602 of Proceedings of SPIE, pp. 194-201, San Jose, Calif, USA, January 1999.

[27] H. Fang, G. Kaur, and B. Wang, "Progress in boronic acidbased fluorescent glucose sensors," Journal of Fluorescence, vol. 14, no. 5, pp. 481-489, 2004.

[28] T. Kawanishi, M. A. Romey, P. C. Zhu, M. Z. Holody, and S. Shinkai, "A study of boronic acid based fluorescent glucose sensors," Journal of Fluorescence, vol. 14, no. 5, pp. 499-512, 2004.

[29] J. C. Pickup, F. Hussain, N. D. Evans, O. J. Rolinski, and D. J. S. Birch, "Fluorescence-based glucose sensors," Biosensors and Bioelectronics, vol. 20, no. 12, pp. 2555-2565, 2005.

[30] D. B. Cordes, S. Gamsey, and B. Singaram, "Fluorescent quantum dots with boronic acid substituted viologens to sense glucose in aqueous solution," Angewandte Chemie International Edition, vol. 45, no. 23, pp. 3829-3832, 2006.

[31] D. B. Cordes, A. Miller, S. Gamsey, and B. Singaram, "Simultaneous use of multiple fluorescent reporter dyes for glucose sensing in aqueous solution," Analytical \& Bioanalytical Chemistry, vol. 387, no. 8, pp. 2767-2773, 2007.

[32] G. Zenkl, T. Mayr, and I. Klimant, "Sugar-responsive fluorescent nanospheres," Macromolecular Bioscience, vol. 8, no. 2, pp. 146-152, 2008.

[33] S. Chinnayelka and M. J. McShane, "Resonance energy transfer nanobiosensors based on affinity binding between apo-enzyme and its substrate," Biomacromolecules, vol. 5, no. 5, pp. 1657-1661, 2004.

[34] S. D'Auria, P. Herman, M. Rossi, and J. R. Lakowicz, "The fluorescence emission of the apo-glucose oxidase from Aspergillus niger as probe to estimate glucose concentrations," Biochemical and Biophysical Research Communications, vol. 263, no. 2, pp. 550-553, 1999.

[35] S. Chinnayelka and M. J. McShane, "Glucose sensors based on microcapsules containing an orange/red competitive binding resonance energy transfer assay," Diabetes Technology \& Therapeutics, vol. 8, no. 3, pp. 269-278, 2006.

[36] C. M. Wong, K. H. Wong, and X. D. Chen, "Glucose oxidase: natural occurrence, function, properties and industrial applications," Applied Microbiology and Biotechnology, vol. 78, no. 6, pp. 927-938, 2008.

[37] M. J. McShane, J. Q. Brown, K. B. Guice, and Y. M. Lvov, "Polyelectrolyte microshells as carriers for fluorescent sensors: loading and sensing properties of a ruthenium-based oxygen indicator," Journal of Nanoscience and Nanotechnology, vol. 2, no. 3-4, pp. 411-416, 2002.

[38] S. R. Nayak, E. W. Stein, N. Gupta, J. Palmer, and M. J. McShane, "Transport of macromolecules through polyelectrolyte microcapsules-effect of molecular size and shell materials," in Proceedings of the 226th ACS National Meeting, p. 204, PMSE, New York, NY, USA, September 2003.

[39] H. Zhu and M. J. McShane, "Macromolecule encapsulation in diazoresin-based hollow polyelectrolyte microcapsules," Langmuir, vol. 21, no. 1, pp. 424-430, 2005.

[40] H. Zhu, E. W. Stein, Z. Lu, Y. M. Lvov, and M. J. McShane, "Synthesis of size-controlled monodisperse manganese carbonate microparticles as templates for uniform polyelectrolyte microcapsule formation," Chemistry of Materials, vol. 17, no. 9, pp. 2323-2328, 2005.

[41] S. R. Nayak and M. J. McShane, "Encapsulation of peroxidase by polymerizing acrylic acid monomers in "clean" polyelectrolyte microcapsules," Journal of Biomedical Nanotechnology, vol. 3, no. 2, pp. 170-177, 2007.

[42] F. Caruso, C. Sch"uler, and D. G. Kurth, "Core-shell particles and hollow shells containing metallo-supramolecular components," Chemistry of Materials, vol. 11, no. 11, pp. 3394-3399, 1999.

[43] F. Caruso, W. Yang, D. Trau, and R. Renneberg, "Microencapsulation of uncharged low molecular weight organic materials by polyelectrolyte multilayer self-assembly," Langmuir, vol. 16, no. 23, pp. 8932-8936, 2000.

[44] E. Donath, G. B. Soukhorukov, and H. M"ohwald, "Novel selfassembled polyelectrolyte multilayer nano- and microcapsules with tailored properties and functions," in Proceedings of the 219th American Chemical Society National Meeting, pp. U517U517, San Francisco, Calif, USA, March 2000.

[45] G. B. Sukhorukov, E. Donath, S. Moya, et al., "Microencapsulation by means of step-wise adsorption of polyelectrolytes," Journal of Microencapsulation, vol. 17, no. 2, pp. 177-185, 2000.

[46] A. Chaudhary and R. Srivastava, "Glucose sensing using competitive binding assay co-encapsulated in uniform sized 
alginate microspheres," Sensor Letters, vol. 6, no. 2, pp. 253 $260,2008$.

[47] K. E. Sapsford, L. Berti, and I. L. Medintz, "Materials for fluorescence resonance energy transfer analysis: beyond traditional donor-acceptor combinations," Angewandte Chemie International Edition, vol. 45, no. 28, pp. 4562-4589, 2006.

[48] G. B. Sukhorukov, A. A. Antipov, A. Voigt, E. Donath, and H. M"ohwald, "pH-controlled macromolecule encapsulation in and release from polyelectrolyte multilayer nanocapsules," Macromolecular Rapid Communications, vol. 22, no. 1, pp. 4446, 2001.

[49] G. Ibarz, L. D" ahne, E. Donath, and H. M"ohwald, “Controlled permeability of polyelectrolyte capsules via defined annealing," Chemistry of Materials, vol. 14, no. 10, pp. 40594062, 2002.

[50] G. Ibarz, L. D"ahne, E. Donath, and H. M"ohwald, "Resealing of polyelectrolyte capsules after core removal," Macromolecular Rapid Communications, vol. 23, no. 8, pp. 474-478, 2002.

[51] Z. An, H. M"ohwald, and J. Li, "pH controlled permeability of lipid/protein biomimetic microcapsules," Biomacromolecules, vol. 7, no. 2, pp. 580-585, 2006.

[52] D. V. Volodkin, N. I. Larionova, and G. B. Sukhorukov, "Protein encapsulation via porous $\mathrm{CaCo}_{3}$ microparticles templating," Biomacromolecules, vol. 5, no. 5, pp. 1962-1972, 2004.

[53] D. V. Volodkin, A. I. Petrov, M. Prevot, and G. B. Sukhorukov, "Matrix polyelectrolyte microcapsules: new system for macromolecule encapsulation," Langmuir, vol. 20, no. 8, pp. 33983406, 2004.

[54] O. P. Tiourina, A. A. Antipov, G. B. Sukhorukov, N. I. Larionova, Y. Lvov, and H. M"ohwald, "Entrapment of $\alpha$-chymotrypsin into hollow polyelectrolyte microcapsules," Macromolecular Bioscience, vol. 1, no. 5, pp. 209-214, 2001.

[55] O. P. Tiourina and G. B. Sukhorukov, "Multilayer alginate/protamine microsized capsules: encapsulation of $\alpha$ chymotrypsin and controlled release study," International Journal of Pharmaceutics, vol. 242, no. 1-2, pp. 155-161, 2002.

[56] N. G. Balabushevich, G. B. Sukhorukov, and N. I. Larionova, "Polyelectrolyte multilayer microspheres as carriers for bienzyme system: preparation and characterization," Macromolecular Rapid Communications, vol. 26, no. 14, pp. 1168-1172, 2005.

[57] E. W. Stein, D. V. Volodkin, M. J. McShane, and G. B. Sukhorukov, "Real-time assessment of spatial and temporal coupled catalysis within polyelectrolyte microcapsules containing coimmobilized glucose oxidase and peroxidase," Biomacromolecules, vol. 7, no. 3, pp. 710-719, 2006.

[58] S. A. Tikhonenko, E. A. Saburova, A. V. Dubrovskii, L. I. Shabarchina, Y. N. Dybovskaya, and B. I. Sukhorukov, "A technique for incorporating enzymes into polyelectrolyte microcapsules," Glass Physics and Chemistry, vol. 33, no. 3, pp. 287-293, 2007.

[59] D. G. Shchukin, D. A. Gorin, and H. M"ohwald, "Ultrasonically induced opening of polyelectrolyte microcontainers," Langmuir, vol. 22, no. 17, pp. 7400-7404, 2006.

[60] W. Tong, W. Dong, C. Gao, and H. M"ohwald, "Chargecontrolled permeability of polyelectrolyte microcapsules," Journal of Physical Chemistry B, vol. 109, no. 27, pp. 1315913165, 2005.

[61] I. L. Radtchenko, G. B. Sukhorukov, and H. M"ohwald, "A novel method for encapsulation of poorly water-soluble drugs: precipitation in polyelectrolyte multilayer shells," International Journal of Pharmaceutics, vol. 242, no. 1-2, pp. 219-223, 2002.
[62] A. I. Petrov, D. V. Volodkin, and G. B. Sukhorukov, "Proteincalcium carbonate coprecipitation: a tool for protein encapsulation," Biotechnology Progress, vol. 21, no. 3, pp. 918-925, 2005.

[63] D. G. Shchukin, G. B. Sukhorukov, and H. M"ohwald, "Smart inorganic/organic nanocomposite hollow microcapsules," Angewandte Chemie International Edition, vol. 42, no. 37, pp. 4472-4475, 2003.

[64] Y. Wang, A. Yu, and F. Caruso, "Nanoporous polyelectrolyte spheres prepared by sequentially coating sacrificial mesoporous silica spheres," Angewandte Chemie International Edition, vol. 44, no. 19, pp. 2888-2892, 2005.

[65] J. S. Schultz, "Sensitivity and dynamics of bioreceptor-based biosensors," Annals of the New York Academy of Sciences, vol. 506, no. 1, pp. 406-414, 1987.

[66] A. Mills and A. Lepre, "Controlling the response characteristics of luminescent porphyrin plastic film sensors for oxygen," Analytical Chemistry, vol. 69, no. 22, pp. 4653-4659, 1997. 

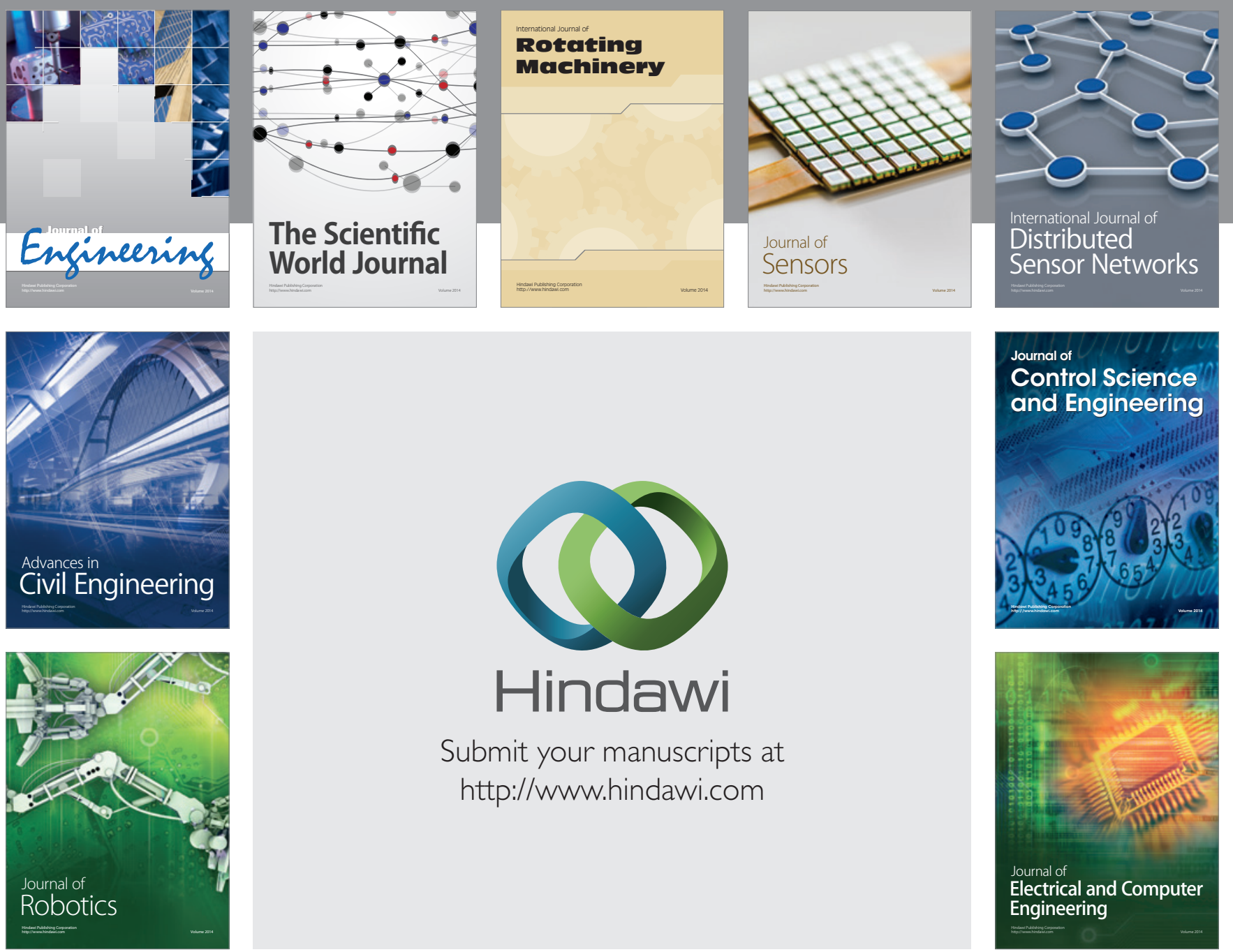

Submit your manuscripts at

http://www.hindawi.com
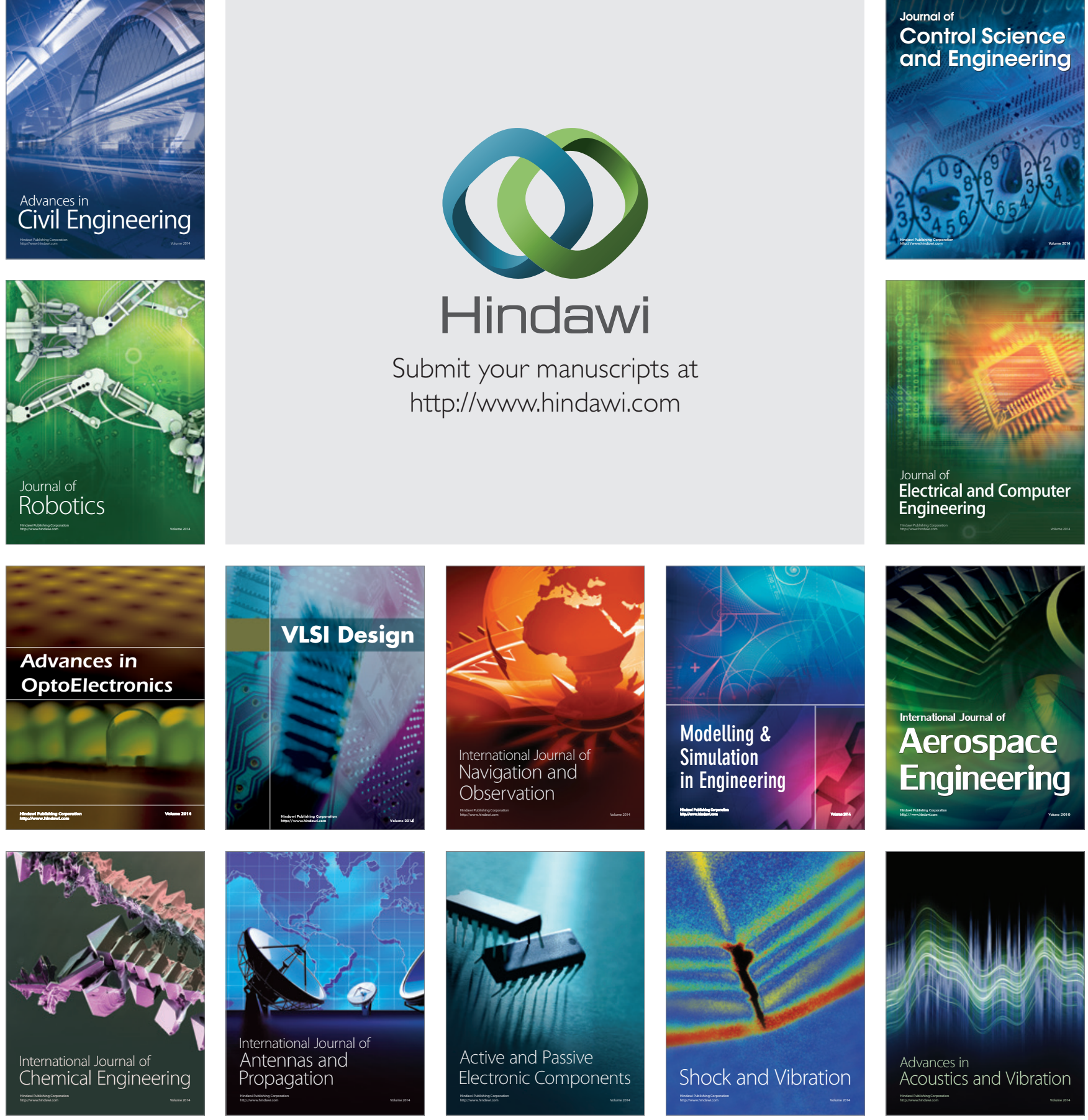